\title{
Sellantes odontopediátricos, ¿Realmente protegen a los dientes de diferentes procesos mecánicos?" Revisión de literatura
}

\author{
Pediatric dentistry sealants, do they really protect teeth from different \\ mechanical processes?" Literature review
}

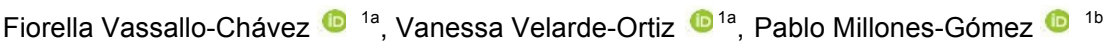

\section{RESUMEN}

El uso de los sellantes de fosas y fisuras en el área de odontopediatría han sido de mucha ayuda como medida preventiva para evitar lesiones cariosas generalmente dados en la zona oclusal de los primeros molares permanentes erupcionados. Estos sellantes han tenido constantes variaciones con el paso del tiempo, sin embargo, los más usados son los sellantes resinosos. Este tipo de sellantes se clasifican principalmente en polimerización, viscosidad y traslucidez, a medida que la odontología ha ido avanzando, estos se dividieron en dos grupos (hidrofóbicos e hidrofílicos), ambos con características diferentes, pero con un mismo propósito, el de brindar una barrera de protección entre factores extrínsecos y el diente. No obstante, al estar en contacto con diferentes elementos como la humedad, carbohidratos y saliva, así como también tener una mala aplicación sobre las fosas y fisuras, estos tienden a fracasar y comienzan a presentar diversos problemas como microfiltración, fallos en la retención y su microdureza. El objetivo de esta revisión de literatura fue reconocer si estos sellantes son los adecuados para proteger los dientes de factores externos y si pueden resistir a diversos cambios mecánicos. Para esto se realizó la revisión bibliográfica en diversas bases de datos y revistas de carácter odontológico, se procedió a la selección de artículos según su evidencia, criterios establecidos y el año de publicación ubicado entre los años 2015 y 2020.

Palabras Clave: Microfiltración; Microdureza, Retención; Sellantes de fosas y fisuras; Hidrofóbico;Hidrofílico. (Fuente: DeCS BIREME)

\section{SUMMARY}

The use of pit and fissure sealants in the area of pediatric dentistry has been very helpful as a preventive measure to avoid carious lesions generally given in the occlusal area of the first permanent molars erupted. These sealants have had constant variations over time, however, the most used are resinous sealants. These types of sealants are mainly classified in polymerization, viscosity and translucency. As dentistry has advanced, they have been divided into two groups (hydrophobic and hydrophilic), both with different characteristics, but with the same purpose, to provide a protective barrier between extrinsic factors and the tooth. However, when in contact with different elements such as humidity, carbohydrates and saliva, as well as having a bad application on the pits and fissures, these tend to fail and begin to present diverse problems such as microfiltration, retention failures and their microhardness. The objective of this literature review was to recognize whether these sealants are adequate to protect the teeth from external factors and whether they can resist various mechanical changes. For this purpose, a literature review was carried out in various databases and dental journals, and articles were selected according to their evidence, established criteria and the year of publication, which was between 2015 and 2020.

Keywords: Microfiltration; Microhardness; Retention; Pit and fissure sealant; Hydrophobic; Hydrophilic. (Source: MeSH NLM)

Recibido: 07 de julio de 2020

${ }^{1}$ Universidad Privada San Juan Bautista. Lima, Perú

${ }^{a}$ Estudiantes de la escuela de Estomatología

${ }^{\mathrm{b}}$ Docente en investigación de la Escuela de Estomatología

Correspondencia:

Pablo Alejandro Millones-Gómez

Dirección: Cesar Vallejo 1429, Lince, Lima -Perú

Correo electrónico: pablodent@hotmail.com
Aprobado: 05 de enero de 2021

Publicado: 08 de abril de 2021

Este es un artículo de acceso abierto distribuido bajo la licencia Creative Commons Atribución 4.0 Internacional (CC BY 4.0) https://creativecommons.org/licenses/by/4.0/deed.es

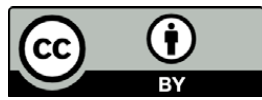

Citar como: Fiorella Vassallo-Chávez, Vanessa Velarde-Ortiz, Pablo Millones-Gómez. Sellantes odontopediátricos, ¿Realmente protegen a los dientes de diferentes procesos mecánicos?” Revisión de literatura. KIRU. 2021 abril-jun-; 18(2): 119- 128 https://doi.org/10.24265/kiru.2021.v18n2.07 


\section{INTRODUCCIÓN}

Siendo la caries una de las enfermedades dentales más predominantes en el mundo y presentándose tanto en niños como en adultos (1), existen diversos métodos de prevención tales como: una buena higiene oral, reducción de azúcares, uso de pre/probióticos, fluoruros ${ }^{(2)}$. Entre ellos también podemos encontrar a los sellantes de fosas y fisuras que han venido siendo utilizados desde mediados de los años $60^{(3)}$.

Estos materiales han sido muy efectivos para prevenir este tipo de lesiones en las diversas superficies de los dientes, ya que forman una barrera protectora para la evitar la invasión de microorganismos y placa ${ }^{(4-6)}$, colocándose generalmente en las fosas y fisuras de las zonas oclusales de los primeros molares (1). Existen diferentes tipos de sellantes, siendo los más usados los que son a base de resina y a base de ionómero de vidrio ${ }^{(6)}$.

Entre las propiedades de estos se debe de encontrar la biocompatibilidad, capacidad de sellado y penetración en la profundidad de fosas y fisuras, además de una capacidad de retención, resistencia al desgaste y ser una opción más económica ${ }^{(3,4)}$.

Enfocándonos en el sellante con base de resina, se tiene conocimiento que el más antiguo NUVASEAL se introdujo en el año 1971 (3). Estos tipos de sellantes dependen generalmente de la retención y del efecto cariostático, entre las desventajas del sellante a base de resina, se atribuye principalmente a la contaminación por humedad de la cavidad bucal ${ }^{(7)}$. Es por ello que en el transcurso de los años se han ido realizando modificaciones químicas a estos sellantes, de los cuales podemos reconocer como sellantes hidrofóbicos e hidrofílicos, en estos dos grupos, diversas marcas comerciales han venido desarrollando nuevas propuestas para su aplicación en boca ${ }^{(5,4)}$.

Sin embargo, podemos reconocer que factores externos tanto como el tiempo de grabado, o inclusive la intensidad de luz LED, aumentan las posibilidades de desarrollar un grado de microfiltración ${ }^{(8,9)}$.

Es por ello que se hizo un análisis a profundidad de diversos artículos que van desde el año 2015 hasta el 2020, con respecto a los sellantes odontopediátricos y se observaron sus capacidades y sus diversos efectos en la microfiltración, microdureza y retención.

\section{MATERIALES Y MÉTODOS}

Se revisaron y recolectaron diferentes artículos científicos provenientes de las bases de datos Medline, Science direct y Google Académico. Para poder encontrar el vocabulario de búsqueda adecuado se utilizó MeSH (acrónimo de Medical Subject Headings) y para su búsqueda en las diversas bases de datos, se utilizó las combinaciones de los conectores Booleanos (AND) (OR) de las palabras a continuación: Microleakage, Microhardness, Retention, Pits and Fissure Sealants, Hidrophobic e Hidrophilic $(($ Microleakage $))$ and ((Pits and Fissure sealants)), ((Microleakage)) or ((Microhardness)) and (( Pits and fissure sealants)), ((Retention)) and ((Pits and fissure sealants)), ((Hydrophilic)) and ((Hydrophobic)) and ((Pits and fissure sealants $))$, ((Hydrophilic)) or ((Hydrophobic)) and ((Pits and fissure sealants $)),(($ Microleakage $))$ and ((Dental sealants))

Los criterios de inclusión instaurados fueron artículos que comparen la microfiltración de los sellantes tanto hidrofílicos e hidrofóbicos; artículos que muestren las diferentes resistencias al sellante; reseñas de diferentes marcas comerciales de sellantes; estudios científicos en laboratorio e in vitro de pruebas de sellantes; artículos con aplicación de grabador y luz led y estudios con respecto a la evolución del sellante bajo el entorno húmedo de la cavidad bucal.

Entre los criterios de exclusión se consideraron artículos que basaban la experiencia en sellantes a base de ionómeros de vidro; los que se referían al uso de agentes de unión para el sellante; estudios con usos de resina fluida usada como sellante de fosas y fisuras y los que involucren microorganismos encontrados en microfiltración. Artículos que no cumplan con los criterios de inclusión.

Un total de 70 artículos científicos, pasaron por un proceso de lectura exhaustiva, primero realizando una revisión detallada de los resúmenes, luego del cuerpo, metodología y conclusiones, seleccionando así solo a aquellos que cumplieran estrictamente los criterios de selección, dando como resultado 37 artículos, para incluirse en esta revision. 


\section{SELLANTES RESINOSOS}

Los sellantes resinosos son aplicados como una barrera física para prevenir la maduración de la dentición recién erupcionada ${ }^{(10)}$, este tipo de sellantes pueden ser clasificados dependiendo del método de polimerización, viscosidad y traslucidez.

Con respecto a la polimerización podemos encontrar cuatro tipos de generaciones:

- Primera generación: El proceso de polimerizado comienza cuando los rayos UV inician dentro del sellante, sin embargo, este proceso ya no es muy usado.

- Segunda generación: Sellantes autopolimerizables y sellantes químicamente curados; el activador una amina terciaria es añadida a sus componentes y mezclada también con otra, la reacción entre estos dos produce radicales libres que producen la polimerización (1-2 minutos de tiempo de espera)

- Tercera generación: Sellantes resinosos con polimerización de luz; la fotopolimerización comienza cuando las longitudes de onda de la lámpara oscilan en d470 nm (azul) y están en contacto con el sellante a base de resina (10-20 segundos de tiempo de espera).

- Cuarta generación: Sellantes resinosos que liberan flúor; las partículas que liberan flúor son añadidas a la generación de materiales anterior con un intento de inhibir lesiones cariosas.

Con respecto a la viscosidad encontramos dos categorías:

- Con relleno: La implementación de partículas de relleno a los sellantes de fosas $y$ fisuras parecen tener un pequeño efecto clínico, adicionalmente estos sellantes con relleno tienen una alta resistencia al desgaste y su habilidad de penetracion a las fisuras es baja.
Ademas los sellantes con relleno suelen requerir ajustes oclusales, lo que alarga el procedimiento.

- Sin relleno: Los sellantes resinosos sin relleno tienen una menor viscosidad, lo cual ofrece una mayor penetración a las fisuras. También permite una mejor retención y rangos de microfiltración mas bajos. Los ajustes oclusales no son necesarios (este procedimiento requiere menos tiempo y el costo es más bajo).

Con respecto a la translucidez tenemos dos categorías:

- Opaco: Los sellantes de fisuras opacos son más fáciles de ver en la aplicación y también más fáciles de detectar en la examinación clínica.

- Transparentes: Los sellantes pueden ser incoloros, rosados o color ambar. (11)

\section{HIDROFÓBICOS E HIDROFÍLICOS}

Dentro de los sellantes resinosos podemos encontrar dos categorías con características diferentes, entre ellos encontramos a los sellantes resinosos hidrofóbicos e hidrofílicos:

Se podría decir que los sellantes de fosas y fisuras Hidrofóbicos son los que mayormente encontramos en el mercado, este tipo de materiales se adhieren a la superficie del esmalte a través de entrelazados micromecánicos. Estos sellantes pueden ser comparables con los de lonómero de vidrio debido a que ambos son retentivos y realizan efectos de remineralización en fosas y fisuras por lesiones cariosas ${ }^{(10)}$, sin embargo, la presencia de la contaminación salival durante su colocación compromete la calidad de unión esmalte-sellante, lo cual es favorable para la microfiltración de microorganismos ${ }^{(12)}$, de igual manera los grados de pérdida de retención mas altos ocurren en la región distal ${ }^{(13)}$.

En algunos estudios científicos lo resultados mostraron que sellantes hidrofóbicos como Clinpro y el Ultraseal Xt Plus tienen una alta adherencia al esmalte ubicado en las fisuras. Clinpro al utiliarse en condiciones 
tanto húmedas como secas proporciona un buen resultado (14) y presenta un efecto preventivo contra las caries $(15,16)$. Otros estudios comentan que el Ultraseal Xt Plus presenta resultados desfavorables por contaminación de saliva en cavidad ${ }^{(14)}$.

Los sellantes Hidrofílicos por su parte tienen la habilidad de proporcionar la transferencia de iones mientras mantienen sus propiedades físicas con la capacidad de proveer un potencial para mejorar los resultados con pacientes. También pueden ofrecer una mayor retención y mejores resultados con los sellantes ya que al ser materiales activos serán beneficiosos en la maduración de los dientes recién erupcionados y serán menos sensitivos que las resinas típicas en la cavidad oral ${ }^{(10)}$. Este tipo de material se adhiere efectivamente en las superficies del esmalte húmedo, lo cual presenta una gran ventaja en la odontología pediátrica, por la complicación en los pacientes con respecto al asilamiento y el control de la humedad (12). Sin embargo, este tipo de sellantes se pueden ver afectados en las regiones distales en aspectos retentivos cuando el aislamiento no es realizado ${ }^{(13)}$.

Se ha demostrado en artículos que el sellante hidrofílico Embrace Wetbond es exitoso en el aspecto de retención (13). En otros estudios Embrace WetBond y UltraSeal XT Hydro presentan resultados variables, como por ejemplo que en ambientes secos demuestra menos formación de gaps, sin embargo, el Embrace Wetbond puede no adaptarse a la superficie $y$ presentar problemas en la adhesión (14) en otras investigaciones este mismo sellante presentó una mejor retención después de 6 meses de seguimiento ${ }^{(10)}$.

\section{MICROFILTRACIÓN}

La microfiltración es la que perjudica al factor de fallo de adherencia entre el sellador y el armazón del diente y puede ser determinada por muchos técnicas in vitro. Con las ventajas de la fiabilidad, simplicidad y facilidad de aplicación, la penetración del colorante es un método bien establecido y comúnmente utilizado para la determinación de microinfiltración in vitro ${ }^{(17)}$. Es definida como el paso indetectable(clínicamente) de bacterias, fluidos, moléculas o iones entre las paredes de la cavidad y el material restaurativo aplicado, como resultado de diferencias en el coeficiente de expansión térmica de material y el tejido dentario o por contracción promovida durante la polimerización $^{(18)}$.

Nogales et al. definieron a la microfiltración como la fuerza de adhesión que supera las fuerzas generadas por la contracción de polimerización y las fuerzas generadas por los cambios dimensionales térmicos posteriores a la polimerización. La microfiltración puede definirse como los espacios largos mediante los cuales hay el paso indetectable de bacterias, fluidos, moléculas e iones entre la pared cavitaria y el material de restauración, esto generalmente es causado cuando la resistencia a la unión es más baja que el estrés de contracción de una restauración ${ }^{(19)}$.

Lussi y Duangthip mostraron que el uso de un agente de unión en situaciones donde hay contaminación de la saliva, es beneficioso para la disminución de microfiltración y también puede aumentar la retención de sellante ${ }^{(20)}$

\section{RETENCIÓN}

Es la permanencia del sellante en la cara oclusal. La evaluación clínica de la retención de los sellantes y fisuras se realiza pasando la punta del explorador por la superficie oclusal valorando su integridad. Si la punta del explorador presenta ausencia de retención, se coloca número uno, que significa que el sellador está totalmente retenido; si el sellador está parcialmente retenido y la punta presenta retención en una zona se coloca número dos y finalmente si el sellador se encuentra completamente perdido, se coloca número tres.

En 2015 Buonocuore, observó después de dos años de la aplicación de un sellante de fotopolimerización, que se redujo la caries en un $99 \%$ en dientes permanentes y $88 \%$ en dientes temporales. En relación a la retención del material, fue del $87 \%$ y $50 \%$ para dientes permanentes y temporales, respectivamente (21)

\section{Evaluación de retención}

En el estudio de Prado et al. ${ }^{(22)}$ Clasificó el estado del sellador de la siguiente manera: retención completa (todas las fosas y fisuras llenas de material), retención parcial (pérdida de algún material) o pérdida completa (ausencia de material de toda la superficie oclusal. 
La efectividad de los selladores a base de resina depende principalmente de la retención $y$, en segundo lugar, del efecto cariostático del sellador. La falla de la retención a base de resina sellante se atribuye principalmente a la contaminación por humedad. Para superar este inconveniente, se ha introducido UltraSeal XT® Hydro, un sellador innovador que es amigable con la humedad, pero no susceptible a la absorción y degradación del agua como otros selladores hidrofílicos ${ }^{(10)}$.

\section{MICRODUREZA}

La microdureza proporciona al sellante una medida de resistencia a la deformación plástica bajo fuerzas compresivas aplicadas ${ }^{(12)}$. La dureza es una propiedad que proporciona una medida relativa de su resistencia a la indentación al aplicar una carga determinada y continua para soportar la indentación o a su vez el rayado, La dureza superficial se determina a la resistencia a la indentación o penetración que tiene un material. Entre sus principales tipos de dureza tenemos las los siguientes:

\section{Dureza Knoop}

Se realiza utilizando un indentador de diamante que posee una forma piramidal; al ser impactada su muesca determina una carga en el material en forma de romboidal en un tiempo establecido por el investigador, se utiliza un microscopio para dichas mediciones; y su indentación es el resultante de un valor de dureza.

\section{Dureza Vickers}

Se utiliza una maquina calibrada para emplear una carga compresiva predeterminada, con un indentador piramidal de diamante con base cuadrada se aplica perpendicularmente al material que se va a medir, con una carga determinada donde se va mantener un tiempo específico y luego es retirada del sitio y se puede observar una medida diagonal en la superficie del material ${ }^{(23)}$.

\section{FACTORES EXTERNOS}

El principal factor de adhesión de un sellante resinoso de fosas y fisuras al esmalte se da por la formación de tags entrelazados micromecanicamente que penetran las macro y micro porosidades presentadas en el esmalte preacondicionados. Existe pequeñas interacciones psico químicas entre la superficie alta de energía de la hidroxiapatita en el esmalte y los constituyentes orgánicos del sellante presente, estos sellantes están diseñados para la colocación de ácido previo (12).

El uso del ácido grabador como paso previo a la colocación de sellantes de fosas y fisuras incrementan la retención e integridad marginal (16). Sin embargo, en el caso de la colocación de sellantes, los restos y la película podrían no ser eliminados de la base de las fracturas mediante los procedimientos convencionales de profilaxis y grabado, es por ello que se propusieron diferentes alternativas para preparar las fisuras con métodos como la abrasión por aire o también láser ${ }^{(24)}$.

La anatomía oclusal de las fosas y fisuras es generalmente retentiva siendo áreas de acumulo de carbohidratos y también de película salival, es por ello que para evitar la penetración a estas es recomendable la profilaxis previa a la colocación del sellante. La perdida de los selladores se puede atribuir al coeficiente de expansión térmica ${ }^{(3)}$, esto se puede relacionar a que en la cavidad oral el $\mathrm{PH}$ cambia constantemente ${ }^{(25)}$.

La alta tasa de la retención en los dientes de la mandíbula se consideraba que se debe a que la aplicación fue fácil, y que los molares mandibulares tienen fisuras bien limitadas. Además, como el maxilar los dientes están expuestos a la tensión oclusal antes que la mandíbula dientes, esto podría contribuir a un bajo nivel de retención ${ }^{(13)}$.

El tiempo de la colocación de los sellantes es crítico debido a que estos dientes recién erupcionados son más susceptibles al desarrollo de las lesiones cariosas es por ello que es recomendable la colocación de los sellantes lo más pronto posible ${ }^{(26,13)} \mathrm{Y}$ se tiene que tener en cuenta la prevención al colocar estos materiales para evitar su contaminación y futuro fracaso ${ }^{(10)}$. 
Tabla 1 Estudios sobre sellantes odontopediatricos (2015-2020)

\begin{tabular}{|c|c|c|c|}
\hline Artículo & Autor & $\begin{array}{c}\text { Año de } \\
\text { Publicación }\end{array}$ & Conclusión \\
\hline $\begin{array}{l}\text { Meta-analysis of the longevity of } \\
\text { commonly used pit and fissure } \\
\text { sealant materials }\end{array}$ & $\begin{array}{l}\text { Jan Kühnisch, Ahmed } \\
\text { Bedir, } \\
\text { Yi-Fang Lo, Andreas } \\
\text { Kessler and et all } \\
\end{array}$ & 2020 & $\begin{array}{l}\text { Los sellantes fotopolimerizables continúan } \\
\text { siendo los referentes para sellantes de fosas y } \\
\text { fisuras }\end{array}$ \\
\hline $\begin{array}{l}\text { Comparative Evaluation of the } \\
\text { Viscosity and Length of Resin } \\
\text { Tags of Conventional and } \\
\text { Hydrophilic Pit and Fissure } \\
\text { Sealants on Permanent } \\
\text { Molars: An In vitro Study }\end{array}$ & $\begin{array}{l}\text { Prabakar J, John J, } \\
\text { Arumugham IM, Kumar RP, } \\
\text { Sakthi DS }\end{array}$ & 2018 & $\begin{array}{l}\text { El sellador hidrofílico (UltraSeal XT Hydro) fue } \\
\text { menos viscoso, lo que resultó una mejor } \\
\text { retención del sellador en comparación del } \\
\text { sellante convencional (Clinpro 3M ESPE). } \\
\text { Además, con el sellador hidrofílico Ultraseal XT } \\
\text { Hydro demostró una ventaja en dientes } \\
\text { parcialmente erupcionados debido a problemas } \\
\text { de control de humedad }\end{array}$ \\
\hline $\begin{array}{l}\text { Retention and remineralization } \\
\text { effect of moisture tolerant resin- } \\
\text { based } \\
\text { sealant and glass ionomer sealant } \\
\text { on non-cavitated pit and fissure } \\
\text { caries: } \\
\text { Randomized controlled clinical trial }\end{array}$ & $\begin{array}{l}\text { Laith Alsabeka } \\
\text {, Zuhair Al-Nerabieahb } \\
\text {, Nada Bsharab } \\
\text {, John C. Comis }\end{array}$ & 2019 & $\begin{array}{l}\text { Embrace WetBond mostró superioridad sobre el } \\
\text { sellador de ionómero de vidrio probado en } \\
\text { retención después de seis meses de } \\
\text { seguimiento }\end{array}$ \\
\hline $\begin{array}{l}\text { Characterisation and } \\
\text { microleakage of a new hydrophilic } \\
\text { fissure sealant ultraSeal XT hydro }\end{array}$ & $\begin{array}{c}\text { Zeynep A, Nazmiye D, and } \\
\text { et al }\end{array}$ & 2016 & $\begin{array}{l}\text { El pre-acondicionamiento con láser disminuye } \\
\text { significativamente la microfiltración y aumenta } \\
\text { la rugosidad de la superficie del esmalte, lo que } \\
\text { provoca la división en zonas de las partículas } \\
\text { de relleno en la interfaz del esmalte-sellador. }\end{array}$ \\
\hline $\begin{array}{l}\text { Evaluación bajo microscopio } \\
\text { electrónico de barrido } \\
\text { de sellantes de fosas y fisuras } \\
\text { hidrófobos e hidrófilos, } \\
\text { bajo diferentes condiciones de } \\
\text { humedad: Un estudio in vitro } \\
\end{array}$ & $\begin{array}{l}\text { Eyra Rangel Padilla, } \\
\text { Hector Ramírez Peña, } \\
\text { Hector Martínez } \\
\text { Menchaca and et al }\end{array}$ & 2018 & $\begin{array}{l}\text { El uso de selladores hidrófilos en cuanto a su } \\
\text { adaptabilidad en un ambiente húmedo, } \\
\text { Embrace WetBond y UltraSeal XT Hydro son } \\
\text { una buena elección, presentando mejores } \\
\text { resultados el UltraSeal XT Hydro. }\end{array}$ \\
\hline $\begin{array}{c}\text { Comparative Evaluation of the } \\
\text { Marginal Sealing Ability of two } \\
\text { Commercially Available Pit and } \\
\text { Fissure Sealants }\end{array}$ & $\begin{array}{l}\text { Lakshmi Priya, Joyson } \\
\text { Moses, Bollam Nammalwar, } \\
\text { and et al }\end{array}$ & 2016 & $\begin{array}{l}\text { Se encontró una diferencia estadísticamente } \\
\text { significativa entre los dos grupos; indicando un } \\
\text { rendimiento mucho mejor de Clinpro en } \\
\text { comparación con Helioseal F. }\end{array}$ \\
\hline $\begin{array}{l}\text { Microleakage of a Self-Adhesive } \\
\text { Flowable Composite, a Self- } \\
\text { Adhesive Fissure Sealant and a } \\
\text { Conventional Fissure Sealant in } \\
\text { Permanent Teeth with/without } \\
\text { Saliva Contamination. }\end{array}$ & $\begin{array}{l}\text { Hosseinipour ZS, Heidari A, } \\
\text { Shahrabi M, } \\
\text { Poorzandpoush K }\end{array}$ & 2019 & $\begin{array}{l}\text { La microfiltración del sellador de fisuras } \\
\text { convencional es menor que la del sellador de } \\
\text { fisuras autoadhesivo y el compuesto } \\
\text { autoadhesivo, independientemente de la } \\
\text { contaminación de la saliva. }\end{array}$ \\
\hline $\begin{array}{l}\text { Retention and caries-preventive } \\
\text { effect of glass ionomer and resin- } \\
\text { based sealants: An 18-month- } \\
\text { randomized clinical trial }\end{array}$ & $\begin{array}{l}\text { Jobair Asma AL ,Hammad } \\
\text { Nouf AL, Alsadhan Salwa, } \\
\text { Salama Fouad }\end{array}$ & 2017 & $\begin{array}{l}\text { Después de } 18 \text { meses, ambos selladores con } \\
\text { liberación de fluoruro (Fuji Triage y Clinpro) } \\
\text { tuvieron retención y efectos preventivos de } \\
\text { caries comparables en niños de } 6 \text { a } 9 \text { años con } \\
\text { riesgo de caries moderado a alto. }\end{array}$ \\
\hline $\begin{array}{c}\text { Retention of pit and fissure sealant } \\
\text { versus flowable } \\
\text { composite: An in vivo one-year } \\
\text { comparative evaluation. }\end{array}$ & $\begin{array}{l}\text { Charanjeet Singh, } \\
\text { Kamalpreet Kaur, Kavisha } \\
\text { Kapoor }\end{array}$ & 2019 & $\begin{array}{c}\text { El composite fluido se retuvo relativamente } \\
\text { mejor que el sellador a los } 12 \text { meses de } \\
\text { seguimiento, aunque los resultados fueron } \\
\text { estadísticamente insignificantes. }\end{array}$ \\
\hline $\begin{array}{l}\text { In Vitro Microleakage Comparison } \\
\text { of Flowable } \\
\text { Nanocomposites and } \\
\text { Conventional Materials Used in Pit } \\
\text { and Fissure Sealant Therapy }\end{array}$ & $\begin{array}{c}\text { Arastoo S, Behbudi A, } \\
\text { Rakhshan V }\end{array}$ & 2019 & $\begin{array}{c}\text { Los compuestos fluidos con nanorelleno } \\
\text { seguido de compuestos fluidos es ventajoso por } \\
\text { la escasa microfiltración después del sellado, } \\
\text { especialmente en comparación con el sellador } \\
\text { a base de resina sin relleno, que podría tener la } \\
\text { mayor microfiltración entre las marcas y tipos } \\
\text { de materiales probados. }\end{array}$ \\
\hline
\end{tabular}




\begin{tabular}{|c|c|c|c|}
\hline $\begin{array}{l}\text { Marginal Microleakage of Conventional } \\
\text { Fissure Sealants and } \\
\text { Self-Adhering Flowable Composite as } \\
\text { Fissure Sealant in } \\
\text { Permanent Teeth }\end{array}$ & $\begin{array}{l}\text { Sara Rahimian Imam, } \\
\text { Nahid Ramazani, } \\
\text { Mohammad Reza Fayazi }\end{array}$ & 2015 & $\begin{array}{l}\text { La microfiltración fue menor usando un } \\
\text { compuesto fluido autoadhesivo en } \\
\text { comparación con el sellador convencional de } \\
\text { fisuras; por lo tanto, el compuesto fluido } \\
\text { autoadhesivo es más efectiva para la terapia } \\
\text { de selladores en pacientes con control difícil } \\
\text { de la saliva }\end{array}$ \\
\hline $\begin{array}{l}\text { In vitro assessment of retention and } \\
\text { microleakage in pit and fissure sealants } \\
\text { following enamel pre etching with sodium } \\
\text { hypochlorite deproteinisation. }\end{array}$ & $\begin{array}{l}\text { A. Garrocho-Rangel, C. } \\
\text { Lozano-Vázquez, C. } \\
\text { Butrón-Tellez-Girón, D. } \\
\text { Escobar-García,S. Ruíz- } \\
\text { Rodriguez, A. Pozos- } \\
\text { Guillén. }\end{array}$ & 2015 & $\begin{array}{l}\text { La retención y la adhesión mejora con } \\
\text { desproteinización por } \mathrm{NaOCl} \text { previa al } \\
\text { acondicionamiendo de ácido grabador. } \\
\text { Además, la microfiltración se reduce } \\
\text { significativamente }\end{array}$ \\
\hline $\begin{array}{l}\text { Ameloplasty is counterproductive in } \\
\text { reducing microleakage around Resin } \\
\text { Modified Glass lonomer and Resin based } \\
\text { fissure sealants. }\end{array}$ & $\begin{array}{l}\text { Tabinda Nawaz Khan, } \\
\text { Farhan Raza Khan, } \\
\text { Syed Yawar Ali Abidi }\end{array}$ & 2020 & $\begin{array}{l}\text { La microfiltración fue significativamente } \\
\text { mayor alrededor de los selladores a base de } \\
\text { RMGIC en comparación con los selladores a } \\
\text { base de resina. La Ameloplastia mostró una } \\
\text { mayor microfiltración en comparación con las } \\
\text { muestras sin ameloplastia, }\end{array}$ \\
\hline $\begin{array}{l}\text { Comparative Evaluation of Hydrophobic } \\
\text { and Hydrophilic Resin-based Sealants: A } \\
\text { Clinical Study }\end{array}$ & $\begin{array}{l}\text { Madhumitha Mohanraj, } \\
\text { Rathna Prabhu, Eapen } \\
\text { Thomas, Senthil Kumar }\end{array}$ & 2019 & $\begin{array}{l}\text { La retención del sellador hidrofóbico (Clinpro) } \\
\text { fue superior a los selladores hidrofílicos } \\
\text { (Embrace Wetbond y Champ). }\end{array}$ \\
\hline $\begin{array}{l}\text { ¿Are self-etch adhesive systems effective in } \\
\text { the retention of occlusal sealants? A } \\
\text { systematic review and meta-analysis }\end{array}$ & $\begin{array}{l}\text { Botton G, , Sonego M, } \\
\text { Munhoz S, Tathiane I. et } \\
\text { al. }\end{array}$ & 2019 & $\begin{array}{l}\text { Los selladores aplicados con sistemas de } \\
\text { self-etch muestran una menor retención a lo } \\
\text { largo del tiempo que los selladores aplicados } \\
\text { en los convencionales, independientemente } \\
\text { del uso de sistemas adhesivos }\end{array}$ \\
\hline
\end{tabular}

Elaborado por: Vanessa Velarde y Fiorella Vassallo

\section{DISCUSIÓN}

Esta revisión de literatura tuvo como propósito poder observar las diversas capacidades de los sellantes resinosos, observar sus diferentes cualidades, así como también indagar un poco más a profundidad si es que estos tipos de sellantes tienen la capacidad necesaria para resistir a la microfiltración, retención, microdureza y si son los ideales para utilizarse en odontopediatría como medida preventiva para evitar lesiones cariosas.

Ahmed y Shukry ${ }^{(3)}$ realizaron una investigación para evaluar la retención de los diferentes tipos sellantes después de un año, es por ello que se dividieron en diversos grupos y se asociaron con un sellante. La evaluación se dio cada 3, 6,9 , y 12 meses y se pudo constatar que los sellantes resinosos mostraron tener un mejor desenvolvimiento que los sellantes a base de ionómero de vidrio. Así mismo, Alsabek et al. (10) en su estudio de remineralización y retención de sellantes de fosas y fisuras, concluyeron que a pesar de las limitaciones del estudio, podian afirmar que para prevenir lesiones cariosas es necesario el sellado de la zona y que esta se puede realizar con el sellante Embrace Wetbond, un sellante de carácter hidrofílico que muestra superioridad en cuestión de retención después de 6 meses de su aplicación.

Si es que hablamos de comparación de sellantes de carácter hidrofóbico e hidrofilico en el estudio de Rangel et al. ${ }^{(14)}$ al hacer la comparación de diferentes marcas comerciales, demostraron que el sellante hidrófilo "Clinpro" en condiciones secas y húmedas tiene buenos resultados al igual que los sellantes hidrófilos "Embrace Wetbond" y "Ultraseal Xt Hydro" que tienen una buena capacidad de adaptación en ambientes húmedos.

Sobre los métodos de uso del ácido grabador Kumar et al. ${ }^{(24)}$ en su investigación realizaron una comparación sobre el uso del láser Er,Cr:YSGG y acido grabador y si es que sus beneficios eran similares, en sus resultados comentaron que el uso de este laser es comparable, en términos de retención y aceptabilidad del paciente con respecto a los sellantes. A diferencia de lo mencionado en el estudio de Güçlü et al. (12) en el que manifiestan que el preacondicionamiento con láser redujo significativamente la 
microfiltración y aumentó la rugosidad de la superficie del esmalte, lo que causó la división en zonas de las partículas de relleno en la interfaz del sellador de esmalte.

La retención del sellante depende en gran medida de la cantidad y calidad del esmalte grabado. Existen numerosos factores que pueden conducir a la falla adhesiva del material, pudiendo ser el componente orgánico (proteínas) de la superficie adamantina, un factor que incida de manera negativa en el acondicionamiento ácido, y, por lo tanto, disminuye la adhesión al sustrato dental. Traslaviña et al. (28) concluyeron que la desproteinización no representa una mejora significativa en la retención del sellador en relación a la técnica convencional de material resinoso sin adhesivo, comparadas en un periodo de 3 y 6 meses.

Villareal Azucena et al. ${ }^{(27)}$ mostraron que la distancia entre la fuente de luz y el material a fotocurar afecta considerablemente la calidad de fotocurado de materiales a base de resina. La profundidad de las fosetas y fisuras varía de 1 a $3 \mathrm{~mm}$. y la altura de las cúspides puede llegar a ser de $3 \mathrm{~mm}$., por lo cual el sellador de fosas y fisuras no podrá ser fotocurado a una distancia menor de $3 \mathrm{~mm}$.

Lakshmi et al. ${ }^{(25)}$ realizan un estudio donde los sellantes de fosas y fisuras son usados para la prevención de caries dental en zonas anatómicamente vulneradas como zonas oclusales con fisuras profundas. El clínico tiene que tener en mente el material con las propiedades mecánicas adecuadas como una buena adhesión, mínima microfiltración que se puede traducir en una eficiencia clínica máxima en los pacientes pediátricos. Akinlotan et al. ${ }^{(37)}$ Hablan sobre el costo efectividad de estos productos dependen del clínico y las condiciones del paciente, se tiene que evaluar marcas comerciales y sus debidas características y aplicación recomendada del fabricante.

Luego de la evaluación de la evidencia mostrada, se concluye que los sellantes resinosos protegen a los dientes de estos factores y que también tenemos que tener en cuenta que al realizar la elección y aplicación de estos, tenemos que realizar pasos previos como la profilaxis y un buen aislamiento para evitar su fracaso. Porque estos sellantes resinosos así sean compatibles con el ambiente húmedo, pueden fracasar en la adhesión y retención.

Contribuciones de autoría: VV, FV y PMG recopilaron, diseñaron el estudio y analizaron los diversos artículos. Redactaron y aprobaron el artículo todos los autores.

Fuente de financiamiento: El estudio fue financiado por los autores

Conflicto de intereses: Los autores manifestaron no tener conflictos de intereses

\section{REFERENCIAS BIBLIOGRÁFICAS}

1. Manjakandy $S$, Santhosh $P$, Tejavathi N, Abdul K, Kumaranachary R. Comparison and Clinical Evaluation of Two Pit and Fissure Sealants on Permanent Mandibular First Molars: An In Vivo Study. J Contemp Dent Pract, 2019, 20(10):1151-1158.

2. Twetman S. Prevention of dental caries as a non-communicable disease. European journal of oral sciences, 2018, 126(1): 1925.

3. Ahmed H, Sukry M. Effectiveness of Seven Types of Sealants: Retention after One Year. Int J Clin Pediatr Dent, 2019, 12(2):96-100.

4. Pritesh G, Vishwas C, Aniey P. Comparison of Microleakage and Penetration Depth between Hydrophilic and Hydrophobic Sealants in Primary Second Molar. Int J Clin Pediatr Dent, 2016, 9(4):291-295.

5. Askarizadeh $\mathrm{N}$, Heshmat $\mathrm{H}$, Zangeneh $\mathrm{N}$. One-Year Clinical Success of Embrace Hydrophilic and Helioseal-F Hydrophobic Sealants in Permanent First Molars: A Clinical Trial.J Dent Tehran, 2017, 14(2):9299.

6. Alkhodairi, A., Alseweed, M., Alwashmi, S., Elmoazen, R. A. Clinical Evaluation of the Retention of Resin and Glass Ionomer Sealants Applied as a Part of School-Based Caries Prevention Program. Open access Macedonian journal of medical sciences, 2019, 7(23): 4127-4130

7. Jayashri P, Joseph J, Meignana A, R Pradeep K, D Srisakthi. Comparative evaluation of retention, cariostatic effect and discoloration of conventional and hydrophilic sealants - A single blinded randomized split mouth clinical trial. Contemp Clin Dent, 2018, 9(1):3-9.

8. Tian, Y. M., Zhuge, R. S., Zhang, Z. T., Zheng, D. X., Ding, N., \& Li, Y. M. Effects of subpressure on the sealing ability of dental sealant in vitro. Journal of the mechanical 
behavior of biomedical materials, 2018, 85:117-123.

9. Bani, M., \& Tirali, R. E. Effect of new light curing units on microleakage and microhardness of resin sealants. Dental materials journal, 2016, 35(3): 517-522.

10. Alsabeka L, Al-Nerabieahb Z, Bsharab N, Comisic J. Retention and remineralization effect of moisture tolerant resin-based sealant and glass ionomer sealant on non cavitated pit and fissure caries: Randomized controlled clinical trial. Journal of Dentistry, 2019, 86(1): 69-74.

11. Colombo S, Beretta M. Dental sealants Part 3: Which material? Efficiency and effectiveness. European journal of paediatric dentistry, 2018, 19(3): 247-249.

12. Güçlü Z, Dönmez N, Coleman N. Characterisation and microleakage of a new hidrophilic fissure sealant- Ultraseal XT Hydro. J Appl Oral Sci, 2016,1(1): 44-51.

13. Topal B, Kirzioğlu Z. Evaluation od the fissure sealants applied to erupting permanente molars in accordance to eruption stages: A prospective study. Nigerian journal of clinical practice, 2019, 22(11): 1495-1502.

14. Rangel E, Ramírez H, Martínez H, Rivera G, Valencia $R$, Lozano M, Martínez $G$, Espinosa R. Scanning electron microscope evaluation of hydrophobic and hydrophilic sealants under different humidity conditions: An in vitro study. Revista de Odontopediatría Latinoamerica, 2018,8(1): 49-65.

15. Al-Jobair A, Hammad A, Alsadhan S, Salama F. Retention and caries-preventive effect of glass ionomer and resin-based sealants: An 18-month-randomized clinical trial. Dental materials journal. 2017, 36(5): 654-661.

16. Madhumitha M, Rathna $P$, Eapen $T$, Senthil K. Comparative Evaluation of Hydrophobic and Hydrophilic resin-based selants: A clinical Study. The journal of contemporary dental practice, 2019, 20(7): 812-817.

17. Hatirli $H$, Yasa B, Yasa E. Microleakage and penetration depth of different fissure sealant materials after cyclic thermo-mechanic and brushing simulation. Dental Materials Journal, 2017, 1(1):1-9.

18. Montes de Oca, S., Morales, C. y Yamamoto, J. A. Valoración de la microfiltración en selladores de fosetas y fisuras empleando la técnica convencional con ácido fosfórico y un sellador con adhesivo autograbable en dientes contaminados con saliva artificial. Revista Odontológica Mexicana, 2015, 14(4):208212
19. Nogales $M$, Soto $P$, Buchi $M$. Sellado marginal en restauraciones indirectas, cementadas con dos sistemas adhesivos diferentes. Rev Clín Periodoncia Implantol Rehabil Oral, 2015, 4(3):106-109.

20. Lussi A, Duangthip D. Microleakage and penetration ability of resin sealant versus bonding system when applied following contamination. Pediatr Dent ,2015 25(1):05-11.

21. Buonocore MG. Caries prevention in pits and fissure with an adhesive resin polymerized by ultraviolet light: a two-year study of a single adhesive application. J Am Dent Assoc, 2015, 82(1):90-93.

22. Prado K. Ribeiro M, Ribeiro J. One year clnical evaluation of the retention of resin and glass ionomer sealant son permanente first molars in children. Braz $\mathrm{J}$ Oral Sci, 2015, 4(3):190-194.

23. Baptista MC, Pagani C, Bottino MC, Benetti R. Una comparación de la microdureza de materiales restaurativos compuestos indirectos. J.Appl. Oral Sci, 2003, 11(2).

24. Kumar G, Dhillon J, Rehman F. A comparative evaluationof retention of pit and fissure sealants placed with Conventional acid etching and $\mathrm{Er}, \mathrm{Cr}$ :YSGG laser etching: A randomised controlled trial. Laser Therapy, 2016, 25(4):291-298.

25. Sridhar L. P, Moses J, Rangeeth BN, \& Sivakumar S. Comparative Evaluation of the Marginal Sealing Ability of two Commercially Available $\mathrm{Pit}$ and Fissure Sealants. Journal of clinical and diagnostic research. JCDR, 2016, 10(9):01-04.

26. Jayashri P, Joseph J, Meignana A, Pradeep $\mathrm{K}$, D.Sri S. Comparative evaluation of the Viscosity and Length of Resin tags of Conventional and hydrophilic pit and fissure sealants on permanents Molars: An in vitro Study. Contemp Clin Dent, 2018; 9(1):38894.

27. Villarreal A, Guerrero J, Yamamoto A, Barceló F. Profundidad de curado de selladores de fosas y fisuras utilizando luz emitida por diodos a diferentes longitudes de distancia. Rev odont mex, 2015, 19(2):76-77

28. Traslaviña J, Sanín I, Zuñiga A. Retención de los selladores de fosas y fisuras con desproteinización del esmalte vs. Técnica convencional. Rev AMOP, 2015, 23(1):2-6.

29. Kühnisch J, Bedir A, Lo YF. Meta-analysis of the longevity of commonly used pit and fissure sealant materials. Dent Mater, 2020, 36(5):158-168.

30. Hosseinipour, Z S, Heidari A, Shahrabi M, \& Poorzandpoush K. Microleakage of a SelfAdhesive Flowable Composite, a SelfAdhesive Fissure Sealant and a 
Conventional Fissure Sealant in Permanent Teeth with/without Saliva Contamination, Frontiers in dentistry, 2019, 16(4): 239-247.

31. Singh, C., Kaur, K., \& Kapoor, K. Retention of pit and fissure sealant versus flowable composite: An in vivo one-year comparative evaluation. Journal of the Indian Society of Pedodontics and Preventive Dentistry, 2019, 37(4): 372-377.

32. Arastoo, S., Behbudi, A., \& Rakhshan, V. In Vitro Microleakage Comparison of Flowable Nanocomposites and Conventional Materials Used in Pit and Fissure Sealant Therapy. Frontiers in dentistry, 2019, 16(1): 21-30.

33. Rahimian-Imam, S., Ramazani, N., \& Fayazi, M. R. Marginal Microleakage of Conventional Fissure Sealants and SelfAdhering Flowable Composite as Fissure Sealant in Permanent Teeth. Journal of dentistry (Tehran, Iran), 2015, 12(6): 430435.
34. Garrocho, A., Lozano C., Butrón C., Escobar D., Ruíz S, Pozos A. In vitro assessment of retention and microleakage in pit and fissure sealants following enamel pre-etching with sodium hypochlorite deproteinisation. European journal of paediatric dentistry, 2015, 16(3): 212-216.

35. Khan N, Khan F, Abidi S. Ameloplasty is counterproductive in reducing microleakage around Resin Modified Glass lonomer and Resin based fissure sealants. Pakistan journal of medical sciences, 2020, 36(3): 544-549.

36. Botton, G, Morgental, C, Scherer, M, Lenzi, $T$, Montagner A, ¿Rocha R. Are self-etch adhesive systems effective in the retention of occlusal sealants? A systematic review and meta-analysis. International journal of paediatric dentistry, 2016, 26(6): 402-411.

37. Akinlotan M, Chen, B, Fontanilla T, Chen A, Fan V. Economic evaluation of dental sealants: A systematic literature review. Com Dent Oral Epid. 2017, 46(1): 38-46

Fiorella Vassallo-Chávez

ORCID https://orcid.org/0000-0002-5721-2798

fiorella.vassallo@upsjb.edu.pe

Vanessa Velarde-Ortiz

ORCID http://orcid.org/0000-0002-2168-1197

vanessa.velarde@upsjb.edu.pe

Pablo Millones-Gómez

ORCID (1) https://orcid.org/0000-0002-7105-0940

pablodent@hotmail.com

Copyright $($ L La revista. La revista Kiru es publicada por la Facultad de Odontología de la Universidad de San Martín de Porres, en Lima, Perú. 